\title{
Comparison of Adjuvant Efficacy of Different Molecular Weights of Chitosan and Aluminum Hydroxide for Inactivated Rabies Vaccine
}

\author{
Amani A. Saleh \\ Veterinary Serum and Vaccine Research Institute; Abassia, Cairo
}

\begin{abstract}
The development of safe, novel adjuvant is necessary to maximize the efficacy of new and/or available vaccines. In this study, three different molecular weights of chitosan (low, medium, high) were evaluated as immunopotentiators/adjuvants of inactivated rabies vaccine in white Norway rats as an experimental model. Two concentrations $(1.5 \% \& 3 \% \mathrm{w} / \mathrm{v})$ of each chitosan type were used in final concentration $5 \mathrm{mg} / \mathrm{ml}$ and $10 \mathrm{mg} / \mathrm{ml}$. Results showed that lymphocyte proliferation were significantly elevated $(P<0.05)$ in all chitosan vaccinated rats compared to aluminum hydroxide gel vaccinated one. All three type of chitosan produced accelerated and enhanced effects on rabies-neutralizing antibody responses in vaccinated groups. Increases in antibody titers together with lymphocytes proliferation responses revealed that chitosan induced both humoral and cell-mediated immune responses. When compared with aluminum hydroxide vaccine adjuvant, chitosan was superior to aluminum hydroxide. The conclusion of these results suggested that chitosan with different $M W$ had a strong potential to increase both cellular and humoral immune responses and that chitosan may be a promising and efficacious adjuvant candidate suitable for inactivated rabies vaccine.
\end{abstract}

Worldwide, classical rabies is one of the most feared zoonoses, devastating and preventable viral disease, poses a serious threat to humans and animals (Liu et al., 2012). The most common form of transmission is through bites from rabid animals. Currently, the most effective way to drastically decrease the infection rates is through veterinary vaccination (Anon, 2010). Fortunately, rabies can be prevented with efficacious inactivated vaccines available for both pre and post exposure (Jackson, 2011).

The rabies vaccines for animals are administered by parenteral or oral route, with inactivated or attenuated rabies virus, and may contain aluminum compounds and saponin as adjuvants. Independent of the vaccine type used, animals have to be vaccinated several times during their lifetime (1-3 years intervals) (Anon, 2011). Experimentally, a large sort of techniques has been applied to improve veterinary rabies vaccine, including new adjuvants (Morei et al., 2004; Ren et al., 2010).

Therefore, improvement of the potency of inactivated vaccines is a basic and necessary requirement to eliminate dog rabies. Two methods for enhancing the potency were commonly used in inactivated rabies vaccine. One approach, increasing the amount and concentration of viral antigen, is costly and usually performed in human rabies vaccines.
Another approach, the addition of immunopotentiators/adjuvants, is generally administered in veterinary biologics (Liu et al., 2012).

Interest to polysaccharides for the development of new adjuvants or immunopotentiators for medical and veterinary vaccines has recently increased due to their many advantages, such as ready availability, low cost, high effectiveness, and low risk of side effects and toxicity. However, the benefits of incorporation of the polysaccharide into inactivated rabies vaccine have been demonstrated (Liu et al., 2012)

Among these polysaccharides, Chitosan is a natural nontoxic biopolymer produced by the deacetylation of chitin, a major component of the shells of crustaceans such as crab, shrimp, and crawfish. Recently, chitosan has received considerable attention for its commercial applications in the biomedical, food, and chemical industries (Baldrick 2010; Heffernanet et al., 2011). In addition to, previous researches employed viscous chitosan solution as an injectable protein delivery system as well as, it extended the subcutaneous residence time of admixed proteins (Heffernan et al., 2011).

Moreover, it is known that polysaccharides including chitosan have weak immunogenic properties. As for chitosan, studies of immunological reactivity after vaccination of 
mice with this adjuvant showed that none of the test animals induced antibodies when evaluated with ELISA methods (VandeVord et al., 2002). However, chitosan has a chemotactic effect on the immune cells, but this effect does not lead to a humoral immune response (Ghendon et al., 2009) and this is one of its important properties making practical application of chitosan as an adjuvant a promising approach.

As the development of safe, novel adjuvants is necessary to maximize the efficacy of new and/or available vaccines, this study was focused on testing of the effectiveness of supporting activity of the chitosan as an adjuvant in association with inactivated rabies vaccine.

\section{Material and methods}

Chitosan. Three different molecular weights (Low, medium and high) of chitosan were purchased from Sigma-Aldrich. It was prepared according to Lubben et al., (2001) by dissolving in $2 \%$ acetic acid. Two concentrations $1.5 \%$ and $3 \%(\mathrm{w} / \mathrm{v})$ from each type of chitosan (low, medium and high $\mathrm{Mw}$ ) were used as described by Zaharoff et al., (2007); Heffernan, et al., (2011).

Rabies vaccine. The Evelyn Rokitniki Abelseth (ERA) strain of rabies virus was supplied by Serum and Vaccine Research Institute, Abbassia, Cairo. The virus was grown on BHK-21 cells, and the infectivity dose $50 \%\left(\mathrm{TCID}_{50}\right)$ was determined (Edries, 1994). Rabies virus (ERA, $10^{6.7} \mathrm{TCID}_{50} / \mathrm{ml}$ ) was inactivated with BEI as described by (Mondal, et al., 2005; Natali, et al., 2005). Each concentration (1.5\% \& 3\%) of chitosan (low, medium, high MW) was added to the inactivated rabies virus suspension in ratio of $1: 2$ at a final concentration of $5 \mathrm{mg} / \mathrm{ml}$ and 10 $\mathrm{mg} / \mathrm{ml}$ (according to previous studies) (Ghendon, et al., 2009).

Aluminum hydroxide gel rabies vaccine. Approximately $90 \mathrm{ml}$ of the inactivated rabies virus was mixed with $10 \mathrm{ml}$ of aluminum hydroxide gel (a final concentration of $0.7 \mathrm{mg} / \mathrm{ml}$ (Liu et al., 2012). This vaccine was used as negative control.

Cell culture. BHK-21 cells were routinely maintained in the laboratory under Dulbecco's Minimum Essential Medium (GIBCO) supplemented with $2 \%$ newborn calf serum, 100 $\mathrm{U} / \mathrm{mL}$ of penicillin $\mathrm{G}$, and $100 \mathrm{~g} / \mathrm{ml}$ of streptomycin and passaged when cells were confluent. It was used in the neutralization test to determine the neutralizing antibody titers.

Challenge virus standard (CVS) strain. The challenge virus standard (CVS) strain of rabies virus was derived from reference stocks held by Serum and Vaccine Research Institute, Abbassia, Cairo. The CVS strain, propagated in mouse brain, was used as challenge virus in vaccine potency test according to Wunderli et al., (2006). Animals and immunization. White Norway rats (4- 6 weeks old) were purchased and randomly divided into seven groups (24rat/group), Gr. (1): vaccinated with LMW chitosan (1.5\%) adjuvanted vaccine. Gr. (2): vaccinated with LMW chitosan (3\%) adjuvanted vaccine. Gr. (3): vaccinated with MMW chitosan (1.5\%) adjuvanted vaccine. Gr. (4): vaccinated with MMW chitosan (3\%) adjuvanted vaccine. Gr. (5): vaccinated with HMW chitosan (1.5\%) adjuvanted vaccine. Gr. (6): vaccinated with HMW chitosan (3\%) adjuvanted vaccine. Gr. (7): vaccinated with aluminum hydroxide gel rabies vaccine. Each rat was immunized with 0.5 $\mathrm{mL}$ of vaccine in hind limb skeletal muscle.

Swiss mice ( $3-5$ weeks old) were randomly split into seven groups (20mice/group). These were used to test the safety and potency of prepared inactivated rabies virus.

All experimental animals were purchased from Serum and Vaccine Research Institute and maintained in a pathogen-free environment with no prior exposure to rabies virus.

\section{Blood samples.}

- Peripheral blood samples were respectively collected from the retro-orbital plexus on days 6 , $13,20,30,40$ and 50 days following vaccination. The cloud-like lymphocyte band was isolated from heparin buff coats by density gradient centrifugation using Ficoll-Pacque (sigma).

- Serum samples were obtained weekly and every 1 month for 6 months. All serum samples were inactivated at $56^{\circ} \mathrm{C}$ for 30 minutes in water bath and kept at $-20^{\circ} \mathrm{C}$ till used for measuring rabies virus neutralizing antibodies.

Safety test. According to Mitchell et al., (1971) the prepared inactivated vaccine was tested for safety by inoculation of a group of suckling mice $(\mathrm{n}=20)$ intracerebrally with $0.03 \mathrm{ml}$ of prepared vaccine without adjuvants. The immunized animals were observed each day for 21 days.

National Institutes of Health (NIH) potency test. The NIH test for rabies vaccine potency was performed as described by Khawplod et al., (2002). Swiss Webster white mice were randomly assigned to five experimental groups in addition to one control group. Five dilutions of the prepared rabies vaccine without adjuvants were prepared, and $0.5 \mathrm{ml}$ was administered 
intraperitoneally on days 0 and 7 . Fourteen days after the last vaccination the immunized animals and a control group of mice were challenged intracerebrally with the $>5$ LD50/dose of challenge virus standard (CVS). The mice were observed for 14 days, rabies-related death or signs were recorded. Deaths occurring within 24 hr of challenge were judged not to be rabiesrelated and were excluded. The starting dilution should optimally protect $85-100 \%$ of the vaccinated mice.

Virus neutralization (VN) test. A rabies virus VN test was carried out in cell culture as described previously by Yoneda et al., (2008). VN antibody (VNA) titer was determined as a reciprocal of the highest serum dilution that inhibited the cytopathic effect in cell culture.

XTT assay. Cell viability was assessed by the nonisotopic XTT assay, as described by the manufacturer (Biomedica, Vienna, Austria). This assay is based on the ability of functional mitochondria in the living cells to convert yellow-colored tetrazolium compound into red formazan. Cellular reduction of the dye was measured using an ELISA reader at $450 \mathrm{~nm}$ (reference $620 \mathrm{~nm}$ ) in which the optical density (OD) reflects the cell viability

Statistics. Data are presented in means \pm SD. Statistical analysis was done by Student's t test and a $\mathrm{P} \leq 0.05$ was considered to be statistically significant.

\section{Results}

Safety and potency (NIH). No clinical signs of rabies disease were demonstrated by direct inoculation of prepared inactivated rabies virus vaccine in mice. The results of NIH potency test showed that vaccinated mice with the starting dilution did not show any clinical signs related to or died from the rabies virus infection until 14 days post challenge (Table \& Fig. 1).

2. Changes in lymphocyte proliferation. The OD450 values of all groups are presented in Table 2, from which lymphocyte proliferation in vaccinated groups was observed to be significantly enhanced in the presence of all the chitosan $(\mathrm{p}<0.05)$. Responses to the low, medium, and high groups differ significantly, but were higher than those to the aluminum hydroxide gel group.

Six days post vaccination, the groups vaccinated with rabies and $L M W$ chitosan showed significantly higher lymphocytes proliferation levels compared to $M M W$ and $H M W$ chitosan. No significant differences were observed between both concentrations $(1.5 \%$ \& $3 \%$ ) in all groups. On d 13, both concentrations of $L M W$ showed significant higher values compared to the other groups with. On d 20,30, and 40 / post vaccination, there was significant difference in the proliferation values among different groups and 3\% chitosan showed higher levels than $1.5 \%$. Decreases in lymphocyte proliferation levels were showed on d 30 post vaccination in alum hydroxide group compared to chitosan that showed diminution values on $\mathrm{d}$ 50 post vaccinations.

Effect of chitosan on rabies-neutralizing antibody titers. Antibody titers were quantified by the VN test as shown in Table 3. Two weeks post immunization, the mean titers of both all chitosan groups were higher than that of the aluminum hydroxide group, and the $1.5 \%$ chitosan groups were lower than those of the 3\% groups. In addition, the all chitosan accelerated the appearance of rabies-neutralizing antibody induced by inactivated rabies vaccines (Fig. 2). The antibody levels of each chitosan group peaked within 6 weeks post vaccination. The antibody titers in the control group decreased more rapidly than in other groups but the responses induced by all $3 \%$ chitosan groups remained highest for at least 5 months. The mean titers of all chitosan groups declined slowly within 6 months post vaccination, and the mean titers of both LMW groups remained the highest till $6^{\text {th }}$ month post vaccination.

Table (1): Potency test of prepared inactivated rabies vaccine 14 days post challenge in mice.

\begin{tabular}{cccc}
\hline Dilution & $\begin{array}{c}\text { Mortality rate } \\
\text { [dead/total (\%)] }\end{array}$ & $\begin{array}{c}\text { Morbidity rate } \\
\text { [Clin.Signs/total (\%)] }\end{array}$ & Protection (\%) \\
\hline $\mathbf{1 ~ ( 1 / 5 )}$ & $0 / 12(0)$ & $0 / 12(0)$ & 100 \\
$\mathbf{2}(\mathbf{1} / \mathbf{2 5})$ & $2 / 11(18)$ & $2 / 11(18)$ & 82 \\
$\mathbf{3 ( 1 / 1 2 5 )}$ & $3 / 11(27)$ & $1 / 11(25)$ & 64 \\
$\mathbf{4 ( 1 / 7 2 5 )}$ & $6 / 12(50)$ & $2 / 12(17)$ & 30 \\
$\mathbf{5 ( 1 / 3 6 2 5 )}$ & $9 / 12(75)$ & $3 / 12(25)$ & 0 \\
Control & $12 / 12(100)$ & $12 / 12(100)$ & 0 \\
\hline
\end{tabular}



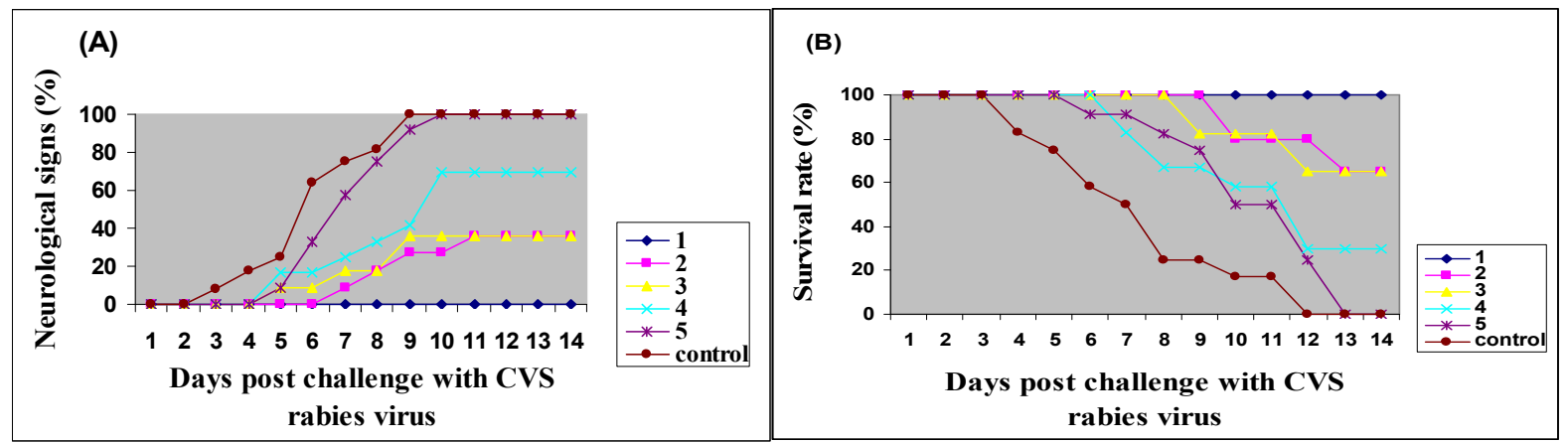

Fig.1 (A and B): Time course of neurological signs and deaths of the mice groups inoculated with the five serial dilutions of prepared inactivated rabies virus post challenge with CVS rabies strain.

Table (2): Mean optical density of Lymphocyte proliferation of rabies vaccine adjuvanted with chitosan.

\begin{tabular}{|c|c|c|c|c|c|c|c|}
\hline \multirow{2}{*}{ Groups } & \multirow{2}{*}{$\begin{array}{l}\text { Type of } \\
\text { vaccine }\end{array}$} & \multicolumn{6}{|c|}{ Days post vaccination } \\
\hline & & 6 & 13 & 20 & 30 & 40 & 50 \\
\hline Gr 1 & $\begin{array}{c}\text { Chitosan } \\
\text { LMW } 1.5 \%\end{array}$ & $1.19 \pm 0.29^{\mathrm{a}}$ & $0.97 \pm 0.03^{\mathrm{a}}$ & $0.63 \pm 0.03^{\mathrm{a}}$ & $0.38 \pm 0.03^{\mathrm{a}}$ & $0.29 \pm 0.04^{\mathrm{a}}$ & $0.18 \pm 0.09$ \\
\hline Gr 2 & $\begin{array}{l}\text { Chitosan } \\
\text { LMW 3\% }\end{array}$ & $1.02 \pm 0.19^{\mathrm{a}}$ & $1.09 \pm 0.13^{\mathrm{a}}$ & $1.69 \pm 0.06^{\mathrm{b}}$ & $0.75 \pm 0.08^{b}$ & $0.46 \pm 0.04^{b}$ & $0.24 \pm 0.26$ \\
\hline Gr 3 & $\begin{array}{l}\text { Chitosan } \\
\text { MMW 1.5\% }\end{array}$ & $0.32 \pm 0.16^{\mathrm{b}}$ & $0.66 \pm 0.02^{\mathrm{b}}$ & $0.89 \pm 0.21^{\mathrm{c}}$ & $0.49 \pm 0.05^{\mathrm{c}}$ & $0.37 \pm 0.03^{\mathrm{c}}$ & $0.12 \pm 0.16$ \\
\hline Gr 4 & $\begin{array}{c}\text { Chitosan } \\
\text { MMW 3\% }\end{array}$ & $0.33 \pm 0.12^{b}$ & $0.88 \pm 0.07^{\mathrm{c}}$ & $0.95 \pm 0.02^{\mathrm{c}}$ & $0.57 \pm 0.07^{\mathrm{c}}$ & $0.34 \pm 0.04^{\mathrm{c}}$ & $0.21 \pm 0.02$ \\
\hline Gr 5 & $\begin{array}{l}\text { Chitosan } \\
\text { HMW } 1.5 \%\end{array}$ & $0.50 \pm 0.02^{\mathrm{c}}$ & $0.42 \pm 0.03^{\mathrm{d}}$ & $0.43 \pm 0.05^{\mathrm{d}}$ & $0.38 \pm 0.03^{\mathrm{d}}$ & $0.32 \pm 0.02^{\mathrm{d}}$ & $0.25 \pm 0.05^{\mathrm{a}}$ \\
\hline Gr 6 & $\begin{array}{l}\text { Chitosan } \\
\text { HMW 3\% }\end{array}$ & $0.60 \pm 0.08^{\mathrm{c}}$ & $0.59 \pm 0.01^{\mathrm{e}}$ & $0.50 \pm 0.01^{\mathrm{e}}$ & $0.47 \pm 0.04^{\mathrm{e}}$ & $0.41 \pm 0.03^{\mathrm{e}}$ & $0.38 \pm 0.03^{b}$ \\
\hline Gr 7 & $\begin{array}{l}\text { Alum as } \\
\text { adjuvant }\end{array}$ & $0.16 \pm 0.01$ & $0.61 \pm 0.03^{f}$ & $0.48 \pm 0.02^{\mathrm{f}}$ & $0.19 \pm 0.03^{\mathrm{f}}$ & $0.19 \pm 0.04^{\mathrm{f}}$ & $0.17 \pm 0.01$ \\
\hline
\end{tabular}

Data within a column with different letters significantly differ $(P<0.05)$.

Table (3): Mean rabies serum neutralization titers in vaccinated groups with inactivated rabies vaccine adjuvated with different molecular weights chitosan.

\begin{tabular}{ccccccccccccc}
\hline \multirow{2}{*}{ Groups } & \multirow{2}{*}{ Type of vaccine } & \multicolumn{10}{c}{ Weeks post vaccination } \\
\cline { 3 - 13 } & $\mathbf{1}$ & $\mathbf{2}$ & $\mathbf{3}$ & $\mathbf{4}$ & $\mathbf{5}$ & $\mathbf{6}$ & $\mathbf{8}$ & $\mathbf{1 2}$ & $\mathbf{1 6}$ & $\mathbf{2 0}$ & $\mathbf{2 4}$ \\
\hline Gr 1 & Chitosan LMW 1.5\% & 16 & 24 & 71 & 84 & 112 & 128 & 112 & 56 & 32 & 27 & 10 \\
Gr 2 & Chitosan LMW 3\% & 32 & 96 & 99 & 123 & 159 & 201 & 126 & 68 & 55 & 40 & 14 \\
Gr 3 & Chitosan MMW 1.5\% & 16 & 32 & 56 & 84 & 128 & 128 & 64 & 56 & 32 & 16 & 4 \\
Gr 4 & Chitosan MMW 3\% & 25 & 56 & 84 & 128 & 128 & 149 & 128 & 64 & 49 & 32 & 8 \\
Gr 5 & Chitosan HMW 1.5\% & 10 & 22 & 32 & 98 & 128 & $\geq 128$ & $>64$ & 50 & $>32$ & 11 & 4 \\
Gr 6 & Chitosan HMW 3\% & 23 & 56 & 85 & 128 & $\geq 128$ & 159 & $\geq 128$ & 98 & 64 & 22 & 8 \\
Gr 7 & Alum as adjuvant & 8 & 12 & 48 & 64 & 64 & 128 & 64 & $\geq 32$ & 16 & 4 & $\geq 2$ \\
\hline
\end{tabular}

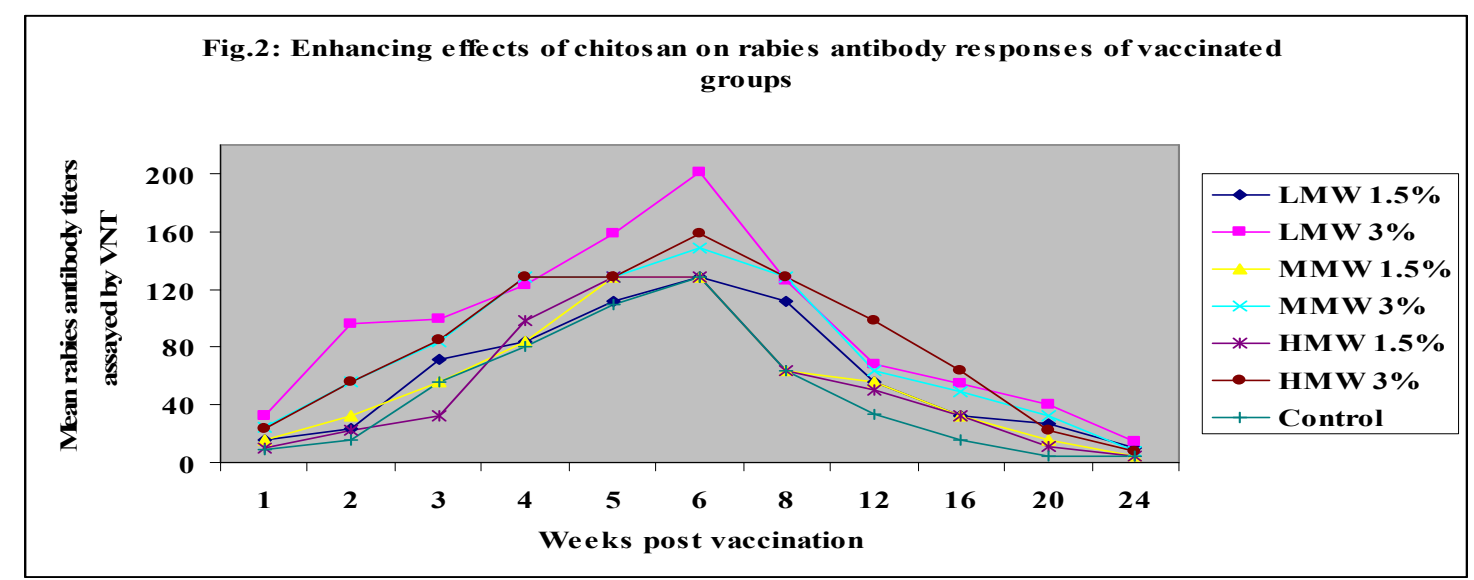




\section{Discussion}

The basic requirements on vaccines applied in human and veterinary immuno-prophylaxis is their safety and protection of human and animal health, especially, their ability to induce an adequate immune reaction capable of ensuring protection against infection. Although a variety of adjuvants have been used in experimental vaccines, most of these materials only elicit an antibody response and/or have undesirable side effects that have limited their potential application in vaccines (Aucouturier, et al., 2001; Hunter, 2002).

The results of NIH potency test showed that none of the mice group of starting dilution showed clinical signs related to or died from the rabies virus infection until 14 days post challenge. This result was in line with Khawplod, et al., (2002) who reported that the starting dilution should optimally protect 85$100 \%$ of the vaccinated mice.

As the cellular immune response plays an important role in the host response to intracellular pathogens by limiting replication and accelerating clearance of infected cells as well as in the generation of both humoral and cell-mediated responses to vaccination (ZhengShun, et al., 2011). Our results obtained by XTT assay revealed that lymphocyte proliferation values (optical density) were significantly higher $(P<0.05)$ all over the experiment in the groups vaccinated with rabies adjuvanted with chitosan compared to the vaccinated group with aluminum hydroxide. Otherwise, these results demonstrated that lymphocytes proliferation values of vaccinated groups with inactivated rabies vaccine adjuvanted with low molecular weight chitosan significantly increased in all vaccinated groups. These increases agreed with previous findings that chitosan exhibited two adjuvant characteristics that were responsible for the enhanced immune response. First, injections of chitosan solution led to a $67 \%$ cellular expansion in local lymph nodes, stimulation of NK (natural killer cells which is the type of cytotoxic lymphocyte that constitute the major component of the innate immune system), in addition to macrophages. The second adjuvant characteristic observed was chitosan's ability to form an antigen depot (Zaharoff et al., 2007) which control the rate of antigen presentation.

The high lymphocytes proliferation activities that persist up to 20 days post vaccination may be revealed to the large difference in viscosities of chitosan solution used in this study led to large differences in antigen dissemination from the injection site and this was came in the same manner with Zaharoff et al., (2007) who discussed that less than $9 \%$ of antigen injected with a saline vehicle remained at the injection site within $8 \mathrm{~h}$. This is contrasted with a chitosan vehicle that maintained a depot of over $60 \%$ injected antigen for 1 week and over $10 \%$ injected antigen for 11 days. Otherwise, the proliferation activities of lymphocytes of chitosan vaccinated groups lasted more than the alum vaccinated group and this attributed to the chitosan can protect the antigen against rapid clearance. The retention of antigen in chitosan creates an ideal scenario for vaccination - a depot of antigen at a site of inflammation that introduces the critical danger signals and costimulation to generate an adaptive immune response (Zaharoff et al., 2007). In comparison between the vaccinated groups, chitosan was found to be superior to aluminum hydroxide. These findings were in agreement with (Gupta, et al., 1995 and Sudheesh, et al., 2011) who recorded that alum salts are relatively poor adjuvants in many situations, particularly at inducing cellular immune responses.

In addition to using different molecular weight chitosans and different concentrations provides tremendous opportunity to engineer chitosan for specific vaccine delivery. Moreover, manipulating the viscosity of chitosan via molecular weight and concentration will change the density of the chitosan matrix and would be expected to control vaccine release and lymphocyte infiltration.

According to previous studies, molecular weights (MW) of chitosan presented an influence on the level of immune response to model antigen when administering with chitosan. However the results of lymphocyte proliferation activities in both MMW\& HMW chitosan vaccinated groups showed relatively decreases compared to LMW groups. As a consequence of increasing MW, some physicochemical and biological properties of chitosan and its solutions change, which determines the bioactivity of the material (Aranaz et al., 2009).

It is known that the ideal adjuvant should also induce both a strong and sustained humoral response with elevated antibody titers as well as a cellular response with memory cells (Cerundolo et al., 2009; Pandey et al., 2009). The rabies-neutralizing anti-body is the most reliable indicator of immune protection. However, alum, the traditional widely-used 
adjuvant, predominantly induces antibody response and cannot meet the requirements (providing rapid and high titers of rabiesneutralizing antibodies) for rabies post-exposure prophylaxis (Y. Liu et al., 2012).

In our study, antibodies titer against rabies virus in all chitosan groups was much higher than alum group and the antibodies levels were remaining through five months post vaccination. This is supported by the results of previous experiments showed that addition of $0.5 \%$ of a chitosan derivative to inactivated influenza vaccine resulted in a 4 to 10 fold increase of antibody titers in parenterally vaccinated mice (Ghendon, et al., 2009).

Moreover, the values resulted by vaccination with $3 \%$ chitosan were higher compared to $1.5 \%$ chitosan. This was attributed to increasing of chitosan viscosity resulting from increased chitosan concentration leading to difference in antigen depot formation and antigen dissemination from the injection site (Zaharoff $e t$ al., 2007)

Previous studies showed that intramuscular administration to mice of inactivated influenza vaccine adjuvanted with a mixture of high- and low- molecular chitosan increased cytotoxic activity of splenic NK T-lymphocytes against the NK-sensitive cell line and proliferative activity of mononuclear lymphocytes in the spleen especially when low- molecular chitosan was used. Moreover, chitosan proliferation activity study suggests that chitosan activates cell immunity and this is an important characteristic of this substance as an adjuvant (Feng et al., 2004; Ghendon, et al., 2009).

Otherwise, a major limitation of alum in vaccine applications is its failure to induce Th1type immune responses. For this reason, alum alone is not a rational choice as an adjuvant for vaccines where a Th1 or mixed Th1/Th2 response is required for protection. This has led to additional research for alternative adjuvants (HogenEsch, 2002). On the other hand, the nature of the immune responses facilitated by chitosan was a mixed Th1/Th2 response (Zaharoff et al., 2007). This was explained the higher results obtained by chitosan adjuvanted vaccine compared to alum hydroxide adjuvanted vaccine.

This finding suggests that chitosan may be used in inactivated rabies vaccines for providing early protection and reduction of rabies antigen dosage. In addition, a rapid cellular response was acquired by the combined use of inactivated vaccine with the polysaccharides, which could be conducive for the development of a new type of therapeutic vaccines. In addition to the high viscosity make it an excellent candidate as a depot/adjuvant for parenteral vaccination alternative to aluminum hydroxide.

\section{References}

Aranaz, I.; Mengíbar, M.; Harris, R.; Paños, I.; Miralles, B.; Acosta, N.; Galed, G. and Heras Á. (2009): Characterization of Chitin and Chitosan Curr Chem Biol, 3: 203-230, PISSN 1872-3136.

Anon (2010): Rabies vaccines: WHO position paperrecommendations. Vaccine 28, (44):7140-2.

Anon (2011): Compendium of animal rabies prevention and control. MMWR Recomm Rep, 60: (RR-6), pp. 1-17

Aucouturier, J.; Dupuis, L. and Ganne, V. (2001): Adjuvants designed for veterinary and human vaccines. Vaccine, 19: 2666-2672.

Baldrick, P. (2010): The safety of chitosan as a pharmaceutical excipient. Regul Toxicol Pharmacol. 56 (3):290-9.

Cerundolo, V.; Silk, J.; Masri, S. and Salio, M. (2009): Harnessing invariant NKT cells in vaccination strategies Nat Rev Immunol, 9 (1) :pp. 28-38

Edries, S. M.; Saber, M. S. and Habashi, Y. Z. (1994): Studies in preparation of inactivated tissue culture antirabies vaccine. Thesis Ph. D. Cairo Uni.

Feng, J.; Zhao, L. and Yu, Q. (2004): Receptor-mediated stimulatory effect of oligochitosan in macrophages. Biochem Biophys Res Commun ;317:414- 20.

Ghendon, Y.; Markushin, S.; Vasiliev, Y.; Akopova, I.; Koptiaeva, I.; Krivtsov, G.; Borisova, O.; Ahmatova, N.; Kurbatova, E.; Mazurina, S. and Gervazieva, V. (2009): Evaluation of properties of chitosan as an adjuvant for inactivated influenza vaccines administered parenterally. J. Med. Virol. 81: 494-506

Gupta, R. K.; Rost, B. E.; Reyveld, E. and Siber, G. S. (1995): Adjuvant properties of aluminum and calcium compounds. In: Powell M F, Newman M J, editors. Vaccine design: the subunit and adjuvant approach. New York, N.Y: Plenum Press, pp. 229-248.

Heffernan, M. J.; Zaharoff, D. A.; Fallon, J. K.; Schlom, J. and Greiner, J. W. (2011): In vivo efficacy of a chitosan/IL-12 adjuvant system for protein-based vaccines. Biomaterials, 32(3): 926-932.

HogenEsch, H. (2002): Mechanism s of stimulation of the immune response by aluminum adjuvants. Vaccine 20(Suppl. 3): S34-S39.

Hunter, R. L. (2002): Overview of vaccine adjuvants: Present and future. Vaccine, 20 (Suppl. 3): S7-S12.

Ren, J.; Sun, L.; Yang, L.; Wang, H.; Wan, M.; Zhang, P.; Yu, H.; Guo, Y.; Yu, Y. and Wang, L. (2010): A novel canine favored $\mathrm{CpG}$ oligodeoxynucleotide capable of enhancing the efficacy of an inactivated aluminumadjuvanted rabies vaccine of dog use Vaccine, 28 (12): pp. 2458-2464

Jackson, A. C. (2011): Therapy of human rabies. Adv Virus Res, 79: pp. 365-375.

Khawplod, P.; Wilde, H.; Tantawicien, T.; Limusanno, S.; Tantawichien, T. and Mitmoonpitak, C. Saikasem, A. and Raksakert, S. (2002): Potency, sterility and immunogenicity of rabies tissue culture vaccine after reconstitution and refrigerated storage for 1 week. Vaccine 20: 2240-2242.

Lubben, I. M.; Verhoef, J. C.; Aelst, A. C.; Borchard, G. 
and Junginger, H. E. (2001): Chitosan microparticles for oral vaccination: preparation, characterization and preliminary in vivo uptake studies in murine peyer's patches.Biomaterials, 22: 687-694.

Mitchell J. R.; Everest R. E. and Anderson, G. R. (1971): Sensitive procedure for detecting residual viable virus in inactivated rabies vaccine. Applied microbiology; 22(4):600-603.

Mondal, S. K.; Neelima, M.; Seetha, R. K.; Ananda, R. K. and Srinivasan, V. A. (2005): Validation of the inactivant binary ethylenimine for inactivating rabies virus for veterinary rabies vaccine production. Biologicals, 33(3): 185-9.

Morein, B.; Hu, K. F. and Abusugra, I. (2004): Current status and potential application of ISCOMs in veterinary medicine. Adv Drug Deliv Rev, 56 (10): 1367-1382.

Natali, F.; Beatriz, A. C.; and Tereza, C. C. (2005): A comparison of serological methods for detecting the immune response after rabies vaccination in dogs and cows from rabies-endemic areas in brazil. Intern J Appl Res Vet Med Vol, No. 3.

Pandey, M.; Batzloff, M.; Good, M. (2009): Mechanism of protection induced by group A Streptococcus vaccine candidate J8-DT: contribution of B and T-cells towards protection. PLoS One, 4 (4), p. e5147.

Sudheesh, M. S.; Vyas, S. P. and Kohli, D. F. (2011): Nanoparticle-based immunopotentiation via tetanus toxoid- loaded gelatin and aminated gelatin nanoparticles. Drug Deliv. Vol. 18(5): 320-330.

VandeVord, P. J.; Matthew, H. W.; DeSilva, S. P.; Mayton, L.; Wu, B. and Wooley, P. H. (2002): Evaluation of the Biocompatibility of a Chitosan Scaffolds in Mice J Biomed Mater Res, 59: 585-590

Wunderli, P. S.; Dreesen, D. W.; Miller, T. J. and Baer, G. M. (2006): The rabies peripheral challenge test: more accurate determination of vaccine potency. Vaccine. 24: 7115-7123.

Liu, Y.; Zhang, S.; Zhang, F. and Hu, R. (2012): Adjuvant activity of Chinese herbal polysaccharides in inactivated veterinary rabies vaccines. I. J Bio Macromol., 50: 598- 602 .

Yoneda, A.; Tuchiya, K.; Takashima, Y.; Arakawa, T.; Tsuji, N.; Hayashi, Y. and Matsumoto, Y. (2008): Protection of mice from rabies by intranasal immunization with inactivated rabies virus. Exp Anim, 57(1): 1-9

Zaharoff, D. A.; Rogers, C. J.; Hance, K. W.; Schlom, J. and Greiner, J. W. (2007): Chitosan solution enhances both humoral and cell-mediated immune responses to subcutaneous vaccination. Vaccine, 25 (11): 2085-94.

Wen, Z.; Xu, Y.; Zou, X. and Xu, Z (2011): Chitosan Nanoparticles Act as an Adjuvant to Promote both Th1 and Th2 Immune Responses Induced by Ovalbumin in Mice. Drugs, 9: 1038-1055. 